NISSUNA UMANA INVESTIGAZIONE SI PUO DIMANDARE VERA SCIENZIA S'ESSA NON PASSA PER LE MATEMATICHE DIMOSTRAZIONI LEONARDO DA VINCI

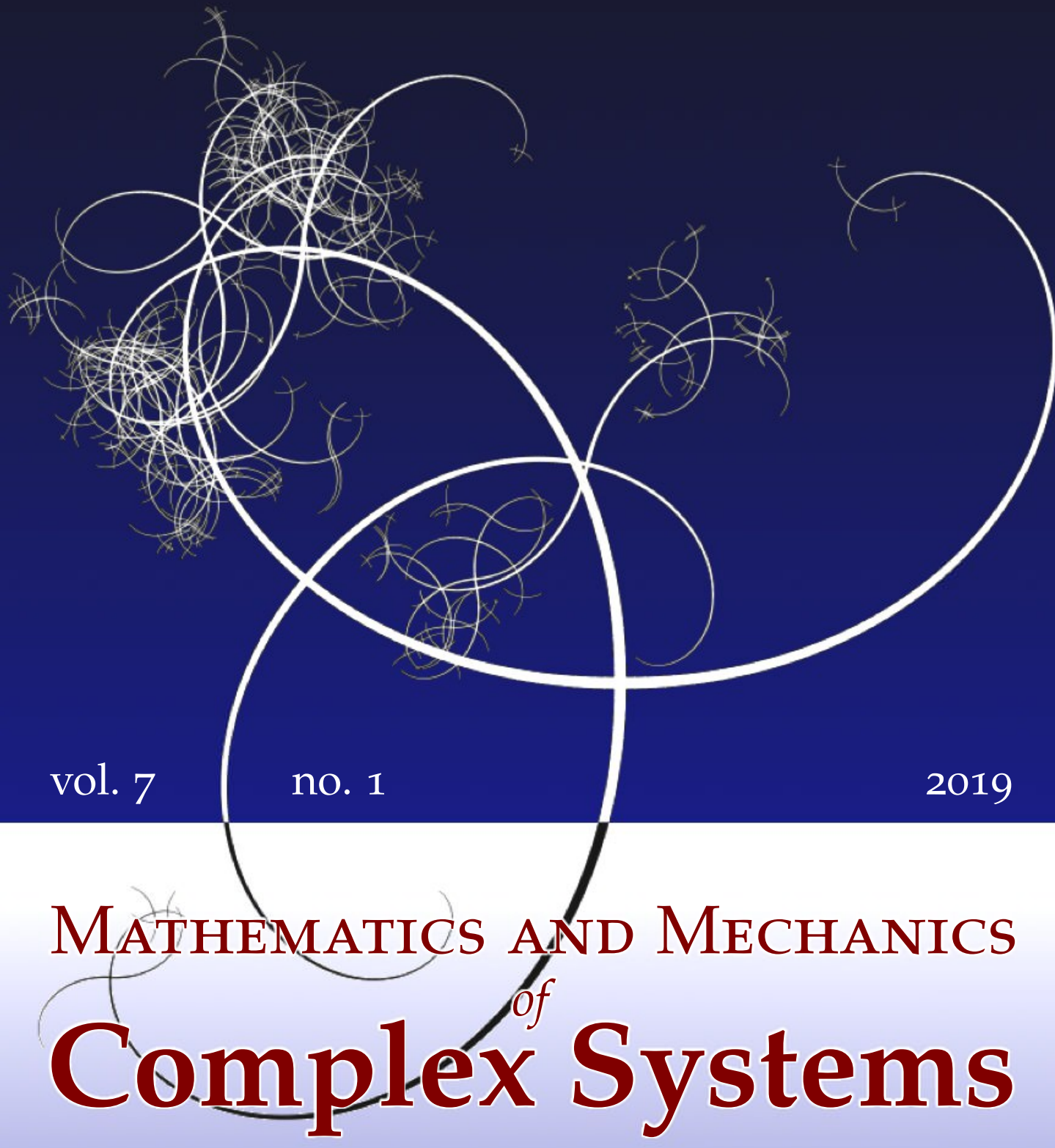

WiLhelm RicKeRT, ELENA N. VilChEVSKAYA AND WOLFGANG H. MÜLLER

A NOTE ON COUETTE FLOW OF MICROPOLAR FLUIDS ACCORDING TO ERINGEN'S THEORY 


\title{
A NOTE ON COUETTE FLOW OF MICROPOLAR FLUIDS ACCORDING TO ERINGEN'S THEORY
}

\author{
Wilhelm RicKERT, ELENA N. VilCHEVSKAYA \\ AND WOLFGANG H. MÜLler
}

\begin{abstract}
In order to model the flow of liquids with internal rotational degrees of freedom the theory of micropolar fluids according to Eringen is applied. The essentials of the theory are outlined and then specialized to Couette flow. The profiles for linear and angular velocities will be computed, and in particular, we shall also study the rise in temperature due to viscous dissipation, which is frequently ignored by mechanicians. Closed-form solutions for all three fields are derived for different boundary conditions. The question as to how the boundary conditions are realized physically will be discussed.
\end{abstract}

\section{Introduction}

This paper is devoted to a description of the flow of fluids with internal rotational degrees of freedom, for example a blood plasma carrying red blood cells or nematic liquid crystals. The flow behavior of such materials can be described by the theory of Eringen (see [Cowin 1974] or [Eringen 2001]). In a nutshell, Eringen's approach, also known as the micropolar theory of fluids, relies on a consistent use of the complete spin balance and the concept of the conservation of microinertia.

Our aim is to study the Couette flow of such fluids not only from the mechanical point of view, i.e., by calculating the linear and angular velocity fields, but also from a thermodynamic one, namely by studying the generation of a temperature field during the flow due to internal dissipation. This will require us to look at the balances of momentum, spin, and internal energy in combination. The fact that, due to the internal dissipation, Couette flow is not isothermal is frequently ignored by the mechanics community. For example, in [Eringen 2001, §9.7], the analysis is explicitly referred to as related to a "steady, isothermal fluid". There are a few papers that address this issue, namely [Kazakia and Ariman 1971; Riha 1975]

\section{Communicated by Francesco dell'Isola.}

Support of this work by a grant from the Russian Foundation for Basic Research (16-01-00815) is gratefully acknowledged.

MSC2010: 74A35, 74A20.

Keywords: polar materials, theory of constitutive functions, closed-form solutions. 
(pressure-driven flow through a plane-parallel channel) and [Singh 1982] (Couette flow). However, in the last reference extremely general boundary conditions are used, which leads to a wealth of constants that make the final solution most difficult to interpret. For such reasons we will be as general as possible but always attempt to give an intuitive interpretation of the results. We will also investigate the boundary conditions, in particular their physical realization, which will hopefully lead to a broader acceptance of micropolar theory in the engineering community. Finally note that for brevity's sake the theoretical part of our presentation will be limited to the absolute essentials.

\section{The relevant balance equations}

Mathematically speaking, our objective is to determine the fields of (a) linear velocity $\boldsymbol{v}(\boldsymbol{x}, t)$, (b) the angular velocity field (also known as microgyration vector [Eringen 2001, p. 8]) $\boldsymbol{\omega}(\boldsymbol{x}, t)$, and (c) the temperature field $T(\boldsymbol{x}, t)$, in all points $\boldsymbol{x}$ and at all times $t$, within a region of space $\mathscr{B}$ through which incompressible matter of constant mass density $\rho_{0}$ and constant (isotropic) microinertia $J_{0}$ is flowing.

The determination of these fields relies on field equations for the primary fields. The field equations are based on balance laws and need to be complemented by suitable constitutive relations later. In spatial description, putting the substantial time derivatives $\frac{\mathrm{d}}{\mathrm{d} t}$ of the balanceable quantities exclusively on the left-hand side, the relevant balances read as

- balance of mass (incompressibility condition)

$$
0=\nabla \cdot \boldsymbol{v}
$$

- balance of linear momentum

$$
\rho_{0} \frac{\mathrm{d} \boldsymbol{v}}{\mathrm{d} t}=\nabla \cdot \sigma
$$

- balance of spin

$$
\rho_{0} J_{0} \frac{\mathrm{d} \omega}{\mathrm{d} t}=\nabla \cdot \boldsymbol{\mu}+\boldsymbol{\sigma}_{\times}
$$

- and the balance of internal energy

$$
\rho_{0} \frac{\mathrm{d} u}{\mathrm{~d} t}=-\nabla \cdot \boldsymbol{q}+\boldsymbol{\sigma}:(\nabla \otimes \boldsymbol{v}+\mathbf{I} \times \boldsymbol{\omega})+\boldsymbol{\mu}:(\nabla \otimes \boldsymbol{\omega}),
$$

where body forces, body couples, and volumetric heat supply have been neglected. The symbol ":" denotes the outer double scalar product $\boldsymbol{C}: \boldsymbol{D}=C_{i j} D_{i j}$. Moreover, $\boldsymbol{\sigma}$ denotes the (nonsymmetric, second-order) Cauchy stress tensor, and $\boldsymbol{\mu}$ is the (second-order) couple stress tensor. In $\boldsymbol{\sigma}_{\times}$the Gibbsian cross, defined by $(\boldsymbol{a} \otimes \boldsymbol{b})_{\times}=$ $\boldsymbol{a} \times \boldsymbol{b}$, is applied to the (nonsymmetric) stress tensor so that its antisymmetric parts are extracted, $u$ is the specific internal energy, and $\boldsymbol{q}$ is the heat flux. For the 
convenience of the reader all tensorial equations are presented in index notation in Appendix A.

\section{Eringen's continuum theory of micropolar fluids}

Eringen's theory of micropolar solids and fluids is summarized comprehensively in [Eringen 1999; 2001], where all of the original literature can also be found. However, for the purpose of this article it is most beneficial to use the condensed version in [Cowin 1974].

The corresponding balance equations of Eringen's micropolar fluid theory are the ones shown in (1)-(4). In [Cowin 1974] a particular focus is on one-dimensional Taylor-Couette and Poiseuille flow problems of an isotropic incompressible micropolar fluid. Hence, the following constitutive equation for the stress tensor is proposed (also see [Zhilin 2012, p. 258]):

$$
\boldsymbol{\sigma}=-p \mathbf{I}+2 \mu \boldsymbol{D}+2 \tau \boldsymbol{h} \times \mathbf{I},
$$

where $p$ is the pressure which in the incompressible case is an arbitrary scalar that guarantees the incompressibility, $\mu$ is the shear viscosity known from classical Navier-Stokes theory, and $\boldsymbol{D}=\frac{1}{2}\left[(\nabla \otimes \boldsymbol{v})^{\top}+\nabla \otimes \boldsymbol{v}\right]$ is the symmetric part of the velocity gradient. The additional antisymmetric part of the stress tensor consists of the (objective) quantity $\boldsymbol{h}=\boldsymbol{\omega}-\boldsymbol{w}$, which is the difference between the angular velocity $\boldsymbol{\omega}$ and the vorticity vector $\boldsymbol{w}=\frac{1}{2} \nabla \times \boldsymbol{v}$, which are both nonobjective. The factor $\tau$ is another viscosity coefficient characteristic of the Cosserat (or antisymmetric) part in the stress tensor. Note that this form of the stress tensor is different from the one in [Cowin 1974]: the terms proportional to $\nabla \cdot \boldsymbol{v}$ are missing due to the incompressibility condition (1). For the couple stress tensor we write

$$
\boldsymbol{\mu}=\alpha \nabla \cdot \boldsymbol{\omega} \mathbf{I}+\beta\left[(\nabla \otimes \boldsymbol{\omega})^{\top}+\nabla \otimes \boldsymbol{\omega}\right]-\gamma\left[(\nabla \otimes \boldsymbol{\omega})^{\top}-\nabla \otimes \boldsymbol{\omega}\right] .
$$

In principle all viscosities $\mu, \tau, \alpha, \beta$, and $\gamma$ can depend upon temperature, but we will ignore this during our analysis. The similarity between the representations of the two stress measures is evident: we are confronted with a linear theory in gradients of velocity and angular velocity ${ }^{1}$ and a linear dependence on the total angular velocity field $\omega$. If these constitutive equations are inserted into the momentum and into the spin balances, (2) and (3), we obtain the coupled system of PDEs

$$
\begin{aligned}
\rho_{0} \frac{\mathrm{d} \boldsymbol{v}}{\mathrm{d} t} & =-\nabla p+(\mu+\tau) \Delta \boldsymbol{v}+2 \tau(\nabla \otimes \boldsymbol{\omega})_{\times}, \\
\rho_{0} J_{0} \frac{\mathrm{d} \boldsymbol{\omega}}{\mathrm{d} t} & =(\alpha+\beta-\gamma) \nabla \nabla \cdot \boldsymbol{\omega}+(\beta+\gamma) \Delta \boldsymbol{\omega}-4 \tau \boldsymbol{\omega}+2 \tau \nabla \times \boldsymbol{v},
\end{aligned}
$$

${ }^{1}$ Recall that $2 \boldsymbol{w} \times \mathbf{I}=(\nabla \otimes \boldsymbol{v})^{\top}-\nabla \otimes \boldsymbol{v}$. 
where $\Delta$ is the Laplace operator. For a derivation as well as for a comparison with the equations in [Cowin 1974], see Appendix A. In order to evaluate the balance of internal energy (4) for the stationary state we need an expression for the heat flux. Following [Eringen 2001, p. 14] we write

$$
\boldsymbol{q}=-\kappa \nabla T+\lambda(\nabla \otimes \omega)_{\times} .
$$

Here $\kappa$ and $\lambda$ are two heat conduction coefficients, the first one being positive and well known from classical Fourier theory. We now proceed to study the solution to the resulting field equations for the case of one-dimensional stationary Couette flow (hence, we do not have to specify a caloric equation of state) between two infiniteplane parallel plates. Observe already now that in this case the non-Fourier part of (8) will play no role.

\section{Specialization to Couette flow}

We shall now concentrate on the solution of a stationary one-dimensional TaylorCouette flow between two parallel infinite plates separated by a distance $h$ in $\boldsymbol{e}_{y^{-}}$ direction [Pennington and Cowin 2000]: possibilities to initiate the motion of the fluid are the following ones. The top plate is subjected to a constant velocity $v_{x}(y=$ $h)=v_{0}$ and provides a constant angular velocity $\omega_{z}(y=h)=\omega_{\mathrm{t}}$, whereas the bottom plate does not move translationally, $v_{x}(y=0)=0$, while an angular velocity $\omega_{z}(y=0)=\omega_{\mathrm{b}}$ is induced. We will refer to such boundary conditions as "velocitycontrolled" or Dirichlet conditions. They are frequently used in the literature, either in this general form [Łukaszewicz 1999] or in a specialized version (e.g., $\omega_{\mathrm{t}}=$ $\omega_{\mathrm{b}}=0$ [Eringen 2001, p. 17] or $\omega_{\mathrm{t}}=-\omega_{0}, \omega_{\mathrm{b}}=\omega_{0}$ [ ̌̌iha 1975; Singh 1982]). Alternatively we may refer to "force-controlled" boundary conditions if a constant shear stress and moment is applied and then transmitted to the fluid, e.g., on the top plate $\sigma_{y x}(y=h)=t_{x}$ and $\mu_{z y}(y=h)=\mu_{z, \mathrm{t}}$ (see [Condiff and Dahler 1964] or [Łukaszewicz 1999, p. 31]). In both cases the fluid will move in the $\boldsymbol{e}_{x}$-direction. We neglect the influence of the specific body force and of the specific body couples and propose the semi-inverse ansatz for the linear and the angular velocities

$$
\boldsymbol{v}=v(y) \boldsymbol{e}_{x}, \quad \boldsymbol{\omega}=\omega(y) \boldsymbol{e}_{z} .
$$

Insertion into the field equations (7) yields the coupled system of ordinary differential equations

$$
0=(\mu+\tau) \frac{\mathrm{d}^{2} v}{\mathrm{~d} y^{2}}+2 \tau \frac{\mathrm{d} \omega}{\mathrm{d} y}, \quad 0=(\beta+\gamma) \frac{\mathrm{d}^{2} \omega}{\mathrm{d} y^{2}}-4 \tau\left(\omega+\frac{1}{2} \frac{\mathrm{d} v}{\mathrm{~d} y}\right) .
$$

Note that there is no contribution from the pressure term in $(7)_{2}$ because we do not apply a pressure gradient in $\boldsymbol{e}_{x}$-direction. We first consider the case of "prescribed 
velocities"; i.e., we impose boundary conditions

$$
v(y=0)=0, \quad v(y=h)=v_{0}, \quad \omega(y=0)=\omega_{\mathrm{b}}, \quad \omega(y=h)=\omega_{\mathrm{t}} .
$$

In view of our interest in the physical realization of boundary conditions, it should be pointed out that the quantities on the right-hand side, i.e., $0, v_{0}, \omega_{\mathrm{b}}$, and $\omega_{\mathrm{t}}$, are kinematic characteristics of the two plates, which must be "transmitted" somehow to the fluid. How this becomes possible is not a question to be asked in context with the mathematical statement. We will get back to it later in the discussion section of the paper.

This system can be solved by rewriting it as a first-order system (see Appendix B). Then the solutions for the linear and angular velocity read

$$
\begin{aligned}
v & =c_{1}+c_{2} h \bar{y}-c_{3} \frac{N h}{L} \exp (2 N L \bar{y})+c_{4} \frac{N h}{L} \exp (-2 N L \bar{y}), \\
\omega & =-\frac{1}{2} c_{2}+c_{3} \exp (2 N L \bar{y})+c_{4} \exp (-2 N L \bar{y})
\end{aligned}
$$

with the dimensionless height $\bar{y}=y / h$ and dimensionless constants $N$ and $L$ defined by

$$
N=\sqrt{\frac{\tau}{\mu+\tau}}, \quad L=\frac{h}{l}, \quad l=\sqrt{\frac{\beta+\gamma}{\mu}} .
$$

By using the boundary conditions (11) the constants $c_{i}$ can be determined:

$$
\begin{aligned}
& c_{1}=-P\left(v_{0}+h \omega^{*}\right) \frac{\mu_{\mathrm{eff}}}{2 \mu}+\frac{h \omega^{\mathrm{d}}}{2} \frac{N^{2}}{P L^{2}}, \quad c_{2}=\left(\frac{v_{0}}{h}+\omega^{*} P\right) \frac{\mu_{\mathrm{eff}}}{\mu}, \\
& c_{3 / 4}=\left(\frac{N}{L} \mp P\right) \frac{\mu_{\mathrm{eff}}}{\mu}\left[ \pm\left(\frac{1}{P}-1\right) \omega^{\mathrm{d}}+\frac{L}{N h}\left(v_{0}+h \omega^{*}\right)\right], \\
& \omega^{*}=\omega_{\mathrm{t}}+\omega_{\mathrm{b}}, \quad \omega^{\mathrm{d}}=\omega_{\mathrm{t}}-\omega_{\mathrm{b}}, \quad P=\frac{N}{L} \tanh (N L), \quad \frac{\mu_{\mathrm{eff}}}{\mu}=\frac{1}{1-P},
\end{aligned}
$$

where $\mu_{\text {eff }}$ is an effective shear viscosity. With the relations

$$
\begin{aligned}
& \frac{\sinh (N L[2 \bar{y}-1])}{\sinh (N L)}=\frac{N}{P L} \sinh (2 N L \bar{y})-\cosh (2 N L \bar{y}), \\
& \frac{\cosh (N L[2 \bar{y}-1])}{\cosh (N L)}=\cosh (2 N L \bar{y})-\frac{P L}{N} \sinh (2 N L \bar{y}),
\end{aligned}
$$


and after some rearrangements the following closed-form solution to the boundary value problem results:

$$
\begin{gathered}
\frac{v(\bar{y})}{v_{0}}=-\frac{P}{2} \frac{\mu_{\mathrm{eff}}}{\mu}\left(\frac{\sinh (N L[2 \bar{y}-1])}{\sinh (N L)}+1-\frac{2 \bar{y}}{P}\right) \\
-\frac{P \mu_{\mathrm{eff}}}{2 \mu} \frac{h \omega^{*}}{v_{0}}\left(\frac{\sinh (N L[2 \bar{y}-1])}{\sinh (N L)}+1-2 \bar{y}\right) \\
+\frac{N^{2}}{2 P L^{2}} \frac{h \omega^{\mathrm{d}}}{v_{0}}\left(1-\frac{\cosh (N L[2 \bar{y}-1])}{\cosh (N L)}\right), \\
\frac{\omega(\bar{y})}{v_{0} / h}=\frac{1}{2} \frac{h \omega^{\mathrm{d}}}{v_{0}} \frac{\sinh (N L[2 \bar{y}-1])}{\sinh (N L)}+\frac{1}{2} \frac{\mu_{\mathrm{eff}}}{\mu}\left(\frac{\cosh (N L[2 \bar{y}-1])}{\cosh (N L)}-1\right) \\
+\frac{h \omega^{*}}{v_{0}} \frac{\mu_{\mathrm{eff}}}{2 \mu}\left(\frac{\cosh (N L[2 \bar{y}-1])}{\cosh (N L)}-P\right) .
\end{gathered}
$$

In the special case of antagonistic spin boundary conditions, $\omega_{\mathrm{t}}=-\omega_{0}$ and $\omega_{\mathrm{b}}=\omega_{0}$, i.e., $\omega^{*}=0$ and $\omega^{\mathrm{d}}=-2 \omega_{0}$, the solution can be simplified [Cowin 1974; Singh 1982]:

$$
\begin{aligned}
& \frac{v(\bar{y})}{v_{0}}=\frac{1}{2} \frac{\mu_{\mathrm{eff}}}{\mu}\left[2 \bar{y}-P\left(1+\frac{\sinh (N L[2 \bar{y}-1])}{\sinh (N L)}\right)\right] \\
& \quad-\frac{h \omega_{0}}{v_{0}} \frac{1}{P}\left(\frac{N}{L}\right)^{2}\left[1-\frac{\cosh (N L[2 \bar{y}-1])}{\cosh (N L)}\right], \\
& \frac{\omega(\bar{y})}{v_{0} / h}=\frac{1}{2} \frac{\mu_{\mathrm{eff}}}{\mu}\left[\frac{\cosh (N L[2 \bar{y}-1])}{\cosh (N L)}-1\right]-\frac{h \omega_{0}}{v_{0}} \frac{\sinh (N L[2 \bar{y}-1])}{\sinh (N L)} .
\end{aligned}
$$

It seems natural to interpret the number $l$, which is given by a ratio of the viscosities occurring in the constitutive equations for the Cauchy and for the couple stress tensors, as a length scale characteristic of the particles on the mesoscale. It is therefore also known as the micropolar length scale parameter in the literature; see for micropolar liquids, e.g., [Cowin 1974], and in an analogous manner also for micropolar solids, see [Zueger and Lakes 2016] as a recent example or [Gauthier and Jahsman 1975], where similar parameters were defined. Similarly, the dimensionless number $N$, the so-called coupling coefficient, is customary and, in our opinion, should preferably be used.

Obviously, we have $0 \leq N \leq 1$, where the zero characterizes traditional NavierStokes flow and values that are close to one stand for a strong influence of the antisymmetric part of the stress tensor. Alternatively we may say that if $N$ approaches zero, the impact of the Cosserat term in the Cauchy stress tensor vanishes and the coupling between linear and angular velocity becomes obsolete.

On the other hand, $0 \leq L \leq \infty$, where $L$ is (through $l$ ) defined by a ratio of new viscosity coefficients and the standard shear viscosity. Also recall that the kinetic 


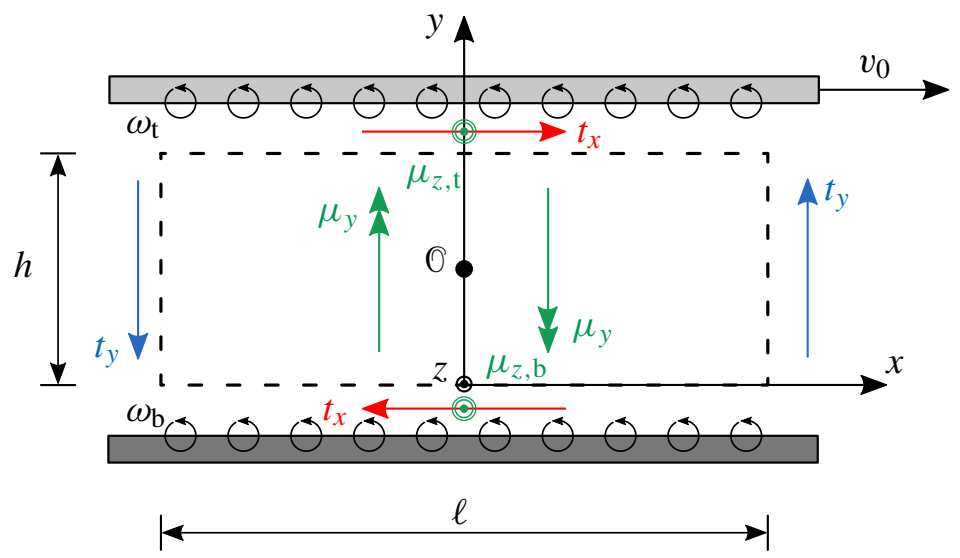

Figure 1. Free body diagram of a section of the Couette flow.

theory of gases allows us to relate the ordinary shear viscosity to the mean free path of molecules [Chapman and Cowling 1952, p. 100]. Therefore, it seems fair to say that $L$-values close to zero indicate a strong influence of characteristic lengths on the mesoscopic scale. In the limiting case where $L$ tends to zero one has

$$
\lim _{L \rightarrow 0} v(\bar{y})=v_{0} \bar{y}+\bar{y}(1-\bar{y}) N^{2} h \omega^{\mathrm{d}}, \quad \lim _{L \rightarrow 0} \omega(\bar{y})=\frac{1}{2}\left(\omega^{*}+\omega^{\mathrm{d}}[2 \bar{y}-1]\right) .
$$

Most interestingly, the linear velocity strongly depends on the boundary conditions of $\omega$, but the angular velocity does not depend on the plate velocity, $v_{0}$. In summary we may say that the Navier-Stokes fluid is obtained if $N \rightarrow 0, L \rightarrow \infty$, and $P \rightarrow 0$. Obviously in this specialized form Eringen's theory has a huge benefit from the viewpoint of the experimentalist: the various new coefficients can be included in only two dimensionless quantities, $N$ and $L$.

It should be noted that Singh [1982] does not define the helpful numbers $L$ and $N$ separately. Rather he combines them in a less intuitive coefficient instead, $\lambda=N L .^{2}$ His solution agrees with ours shown in (15), but it is harder to interpret, in particular, if the intention is not to focus on a special micropolar medium (as Singh does) but to study the impact of the new parameters in general.

We now proceed to discuss the force-controlled experiment. To this end it is useful to consider the free body diagram of the Couette flow shown in Figure 1. Note that the cuts at the upper and lower plates were moved slightly into the fluid region, which enables us to depict the various forces and moments clearly. Of course these cuts are supposed to be flush to the plate surfaces: it is assumed that prescribed forces and moments are transmitted in full from the plate surfaces onto the fluid flanks.

\footnotetext{
${ }^{2}$ Besides that he uses a second coefficient $\xi=2 N^{2}$.
} 


\begin{tabular}{|c|c|c|c|}
\hline flank & $\begin{array}{c}\text { normal } \\
\boldsymbol{n}\end{array}$ & $\begin{array}{l}\text { force traction } \\
\qquad n \cdot \sigma\end{array}$ & $\begin{array}{c}\text { moment traction } \\
\boldsymbol{n} \cdot \boldsymbol{\mu}\end{array}$ \\
\hline $\begin{array}{l}\text { top } \\
\text { bottom }\end{array}$ & $\begin{array}{l}+\boldsymbol{e}_{y} \\
-\boldsymbol{e}_{y}\end{array}$ & $\begin{array}{l}\boldsymbol{t}_{x}=\mu_{\mathrm{eff}}\left(v_{0} / h+P \omega^{*}\right) \boldsymbol{e}_{x} \\
\boldsymbol{t}_{x}=-\mu_{\mathrm{eff}}\left(v_{0} / h+P \omega^{*}\right) \boldsymbol{e}_{x}\end{array}$ & $\begin{aligned} \boldsymbol{\mu}_{z, \mathrm{t}}= & \left(\mu_{\mathrm{eff}} P\left[v_{0}+h \omega^{*}\right]\right. \\
& \left.+\left(\mu N^{2} /\left(P L^{2}\right)\right) h \omega^{\mathrm{d}}\right) \boldsymbol{e}_{z} \\
\boldsymbol{\mu}_{z, \mathrm{~b}}= & \left(\mu_{\mathrm{eff}} P\left[v_{0}+h \omega^{*}\right]\right. \\
& \left.-\left(\mu N^{2} /\left(P L^{2}\right)\right) h \omega^{\mathrm{d}}\right) \boldsymbol{e}_{z}\end{aligned}$ \\
\hline $\begin{array}{l}\text { right } \\
\text { left }\end{array}$ & $\begin{array}{l}+\boldsymbol{e}_{x} \\
-\boldsymbol{e}_{x}\end{array}$ & $\begin{aligned} \boldsymbol{t}_{y} & =\mu_{\mathrm{eff}}\left([1-2 P] v_{0} / h-P \omega^{*}\right) \boldsymbol{e}_{y} \\
\boldsymbol{t}_{y} & =-\mu_{\mathrm{eff}}\left([1-2 P] v_{0} / h-P \omega^{*}\right) \boldsymbol{e}_{y}\end{aligned}$ & $\begin{array}{l}\mathbf{0} \\
\mathbf{0}\end{array}$ \\
\hline $\begin{array}{l}\text { front } \\
\text { back }\end{array}$ & $\begin{array}{l}+\boldsymbol{e}_{z} \\
-\boldsymbol{e}_{z}\end{array}$ & $\begin{array}{l}\mathbf{0} \\
\mathbf{0}\end{array}$ & $\begin{array}{l}\boldsymbol{\mu}_{y}=(\beta-\gamma)\left(\omega^{\mathrm{d}} / h\right) \boldsymbol{e}_{y} \\
\boldsymbol{\mu}_{y}=-(\beta-\gamma)\left(\omega^{\mathrm{d}} / h\right) \boldsymbol{e}_{y}\end{array}$ \\
\hline
\end{tabular}

Table 1. Force and moment tractions on the flanks of the free body without the (constant) pressure contribution.

We now calculate all forces and moments acting on the free body. From (5), (6), and (15) the force and moment tractions, $\boldsymbol{n} \cdot \boldsymbol{\sigma}$ and $\boldsymbol{n} \cdot \boldsymbol{\mu}$, respectively, on the six flanks of the free body rectangular parallelepiped can be obtained. They are indicated in Figure 1 by means of arrows and double arrows pointing in the corresponding directions. Note that the balance of linear momentum implies $p=$ $p(x)$ for the pressure. In the pure Couette flow no pressure gradient in $\boldsymbol{e}_{x}$-direction is applied and hence we conclude that the pressure is a constant. Therefore, we do not mention the pressure in our considerations of the free body diagram since it cancels out in the equilibrium of forces and moments.

A few remarks are in order. Whilst the force tractions on the top and bottom plates are constant, the force tractions of the right and left sides are not. They depend on height $y$ and are given by

$$
\boldsymbol{n} \cdot \boldsymbol{\sigma}(y)= \pm\left[(\mu-\tau) \frac{\mathrm{d} v}{\mathrm{~d} y}-2 \tau \omega\right] \boldsymbol{e}_{y} .
$$

As customary in technical mechanics we replace them by statically equivalent averages located at the center of mass, i.e., at height $y=h / 2$, given by

$$
\boldsymbol{t}_{y}=\frac{1}{h} \int_{0}^{h} \boldsymbol{n} \cdot \boldsymbol{\sigma}(y) \mathrm{d} y,
$$

and this result is shown in Table 1 and in the figure. A similar remark holds for the moment tractions acting on the front and back surfaces:

$$
\boldsymbol{n} \cdot \boldsymbol{\mu}(y)= \pm(\beta-\gamma) \frac{\mathrm{d} \omega}{\mathrm{d} y} \boldsymbol{e}_{y}, \quad \boldsymbol{\mu}_{y}=\frac{1}{h} \int_{0}^{h} \boldsymbol{n} \cdot \boldsymbol{\mu}(y) \mathrm{d} y .
$$


Furthermore, note that by looking at the free body diagram the equilibrium of forces is immediately obvious, whereas equilibrium of moments is evident only for the momentum balance in $\boldsymbol{e}_{y}$ - but not in $\boldsymbol{e}_{z}$-direction. Here we must write ( $d$ being the depth of the channel)

$$
\begin{aligned}
\sum M_{z}^{(\mathcal{O})}= & \left(\mu_{z, \mathrm{t}}+\mu_{z, \mathrm{~b}}\right) \ell d+2 t_{y} h d \frac{\ell}{2}-2 t_{x} \ell d \frac{h}{2} \\
= & 2 \mu_{\mathrm{eff}} P\left(v_{0}+h \omega^{*}\right) \ell d+\mu_{\mathrm{eff}}\left([1-2 P] \frac{v_{0}}{h}-P \omega^{*}\right) h d \ell \\
& -\mu_{\mathrm{eff}}\left(\frac{v_{0}}{h}+P \omega^{*}\right) \ell d h \equiv 0 .
\end{aligned}
$$

Moreover, from Table 1 we conclude that in order to keep the lower and upper plates at constant translational velocities 0 and $v_{0}$, and to ensure constant angular velocities $\omega_{\mathrm{t}}$ and $\omega_{\mathrm{b}}$, we must apply constant horizontal shear-like force tractions $t_{x}$ and two constant out-of-plane moment tractions $\mu_{z, \mathrm{t}}$ and $\mu_{z, \mathrm{~b}}$ as

$$
\begin{aligned}
t_{x} & =\mu_{\mathrm{eff}} \frac{v_{0}}{h}+\mu_{\mathrm{eff}} P \omega^{*}, \quad \mu_{z, \mathrm{t}}=\mu_{\mathrm{eff}} P\left[v_{0}+h \omega^{*}\right]+\frac{\mu N^{2}}{P L^{2}} h \omega^{\mathrm{d}}, \\
\mu_{z, \mathrm{~b}} & =\mu_{\mathrm{eff}} P\left[v_{0}+h \omega^{*}\right]-\frac{\mu N^{2}}{P L^{2}} h \omega^{\mathrm{d}},
\end{aligned}
$$

which can be inverted to represent the boundary conditions from (11)

$$
\begin{aligned}
v_{0} & =\frac{2 h t_{x}-\left(\mu_{z, \mathrm{t}}+\mu_{z, \mathrm{~b}}\right)}{2 \mu}, \\
\omega_{\mathrm{t}} & =\frac{\left(L^{2} P^{2} / N^{2}\right)\left(\mu_{z, \mathrm{~b}}-\mu_{z, \mathrm{t}}\right)-\left(\mu_{z, \mathrm{~b}}+\mu_{z, \mathrm{t}}\right)+2 h P t_{x}}{4 h P \mu}, \\
\omega_{\mathrm{b}} & =\frac{\left(L^{2} P^{2} / N^{2}\right)\left(\mu_{z, \mathrm{~b}}-\mu_{z, \mathrm{t}}\right)+\left(\mu_{z, \mathrm{~b}}+\mu_{z, \mathrm{t}}\right)-2 h P t_{x}}{4 h P \mu} .
\end{aligned}
$$

This gives us the possibility to employ "force-controlled" boundary conditions, $t_{x}, \mu_{z, \mathrm{t}}$, and $\mu_{z, \mathrm{~b}}$, instead of "velocity-controlled" ones, namely $v_{0}, \omega_{\mathrm{t}}$, and $\omega_{\mathrm{b}}$. The "forces" as well as "moments" depend on both the prescribed velocity and the prescribed spin. All of this is compatible with the conviction of Eulerian mechanists according to which the balances of momentum and of total moment of momentum are fully independent and so are the boundary conditions, may they be translational and angular velocities or forces and moments per unit area. The two latter pairs are dual to each other so to speak and, with a grain of salt, we may want to refer to them also as Dirichlet and Neumann conditions, respectively [Łukaszewicz 1999, p. 25 and p. 31]. 
Equation (23) also allows us to adapt the solutions shown in (15) to the forcecontrolled situation easily as

$$
\begin{aligned}
& \frac{v(\bar{y})}{h t_{x} / \mu}=\bar{y}-\frac{\left(\mu_{z, \mathrm{t}}+\mu_{z, \mathrm{~b}}\right)}{4 h t_{x}}(\left.1+\frac{\sinh (N L[2 \bar{y}-1])}{\sinh (N L)}\right) \\
&+\frac{\left(\mu_{z, \mathrm{t}}-\mu_{z, \mathrm{~b}}\right)}{4 h t_{x}}\left(1-\frac{\cosh (N L[2 \bar{y}-1])}{\cosh (N L)}\right), \\
& \frac{\omega(\bar{y})}{t_{x} / \mu}=-\frac{1}{2}+\frac{P L^{2}}{4 N^{2}} \frac{\left(\mu_{z, \mathrm{t}}-\mu_{z, \mathrm{~b}}\right)}{h t_{x}} \frac{\sinh (N L[2 \bar{y}-1])}{\sinh (N L)} \\
&+\frac{1}{4 P} \frac{\left(\mu_{z, \mathrm{t}}+\mu_{z, \mathrm{~b}}\right)}{h t_{x}} \frac{\cosh (N L[2 \bar{y}-1])}{\cosh (N L)},
\end{aligned}
$$

and in the special case of antagonistic spin boundary conditions, i.e., $\omega^{*}=0$, as

$$
\begin{aligned}
& \frac{v(\bar{y})}{h t_{x} / \mu}=\bar{y}-P\left[1+\frac{\sinh (2 N L[\bar{y}-1])}{\sinh (2 N L)}\right]+\frac{\xi}{2}\left[1-\frac{\cosh (N L[2 \bar{y}-1])}{\cosh (N L)}\right], \quad \text { (25) } \\
& \frac{\omega(\bar{y})}{t_{x} / \mu}=-\frac{1}{2}\left[1-\frac{\cosh (2 N L[\bar{y}-1])}{\cosh ^{2}(N L)}\right]+\frac{\xi}{2} \frac{L}{N} \frac{\sinh (N L[2 \bar{y}-1])}{\cosh (N L)}, \quad \xi=\frac{\mu_{z, \mathrm{t}}}{h t_{x}} .
\end{aligned}
$$

They allow us to study what happens if we keep the force or moment tractions, $t_{x}, \mu_{z, \mathrm{t}}$, and $\mu_{z, \mathrm{~b}}$, at a constant level: we expect that the velocities of the upper plate go down if the effective viscosity increases, i.e., if we depart from the case of classical Navier-Stokes. This we shall discuss in the next section.

In the special case $\omega_{\mathrm{t}}=-\omega_{\mathrm{b}}=-\omega_{0}$, and subsequently $\omega^{*}=0$, it is curious that the "force", $t_{x}$ in (22), depends only on $v_{0}$ whereas the "moment", $\mu_{z, t}$, depends on both $v_{0}$ and $\omega_{0}$. In order to achieve a certain top plate velocity whilst the spin on the boundaries is of the same magnitude but of different sign, a traction $t_{x}$ that is similar to the ordinary Navier-Stokes fluid case needs to be applied. The difference from the classical solution lies in the effective shear viscosity which contains many of the new viscosities. For simplicity we will discuss in what follows only the special case of "opposite" spin boundary conditions (as in [Řiha 1975] or [Singh 1982]) so that $\omega^{*}$ vanishes in all further considerations.

A singular case arises if we require "no-slip" boundary conditions for the angular velocity. Then $\omega_{0}=0$ and obviously $\mu_{z, \mathrm{t}}=h P t_{x}$, so that the force seems to dictate the behavior of the moment, which is an unpleasant thought from the viewpoint of Eulerian mechanists. We will get back to this issue in the discussion section of this paper.

We now turn to the task of computing the temperature increase due to stationary viscous flow. The following stationary heat conduction equation results from the energy balance (4) and attains the following form if the ansatz (9), together with 
$T=T(y)$, is used:

$$
0=\kappa \frac{\mathrm{d}^{2} T}{\mathrm{~d} y^{2}}+(\mu+\tau)\left(\frac{\mathrm{d} v}{\mathrm{~d} y}\right)^{2}+4 \tau \omega^{2}+4 \tau \omega \frac{\mathrm{d} v}{\mathrm{~d} y}+(\beta+\gamma)\left(\frac{\mathrm{d} \omega}{\mathrm{d} y}\right)^{2} .
$$

The integration can be performed in closed form. For the boundary conditions we assume that two reservoirs of constant temperature $T_{\text {res }}$ are attached to the upper and lower plates:

$$
\begin{aligned}
\frac{T(\bar{y})-T_{\text {res }}}{T_{\text {ref }}}=\frac{a}{2}\{\bar{y}(1-\bar{y})- & \frac{1}{L^{2}}\left(1-\frac{\cosh (N L[2 \bar{y}-1])}{\cosh (N L)}\right) \\
& \left.-\frac{1}{8 L^{2}} \frac{\cosh (2 N L[2 \bar{y}-1])-\cosh (2 N L)}{\cosh ^{2}(N L)}\right\} \\
& -\frac{b}{4 L^{2}}\left(2 \bar{y}-1-\frac{\sinh (N L[2 \bar{y}-1])}{\sinh (N L)}\right) \\
& -\frac{c}{4 L^{2}} \frac{\cosh (2 N L[2 \bar{y}-1])-\cosh (2 N L)}{\sinh ^{2}(N L)}
\end{aligned}
$$

with the "dimensionless" temperatures

$$
a=\frac{\mu v_{0}^{2}}{\kappa T_{\mathrm{ref}}}\left(\frac{\mu_{\mathrm{eff}}}{\mu}\right)^{2}, \quad b=\frac{\mu v_{0} h \omega_{0}}{\kappa T_{\mathrm{ref}}} \frac{\mu_{\mathrm{eff}}}{\mu}, \quad c=\frac{\mu\left(h \omega_{0}\right)^{2}}{\kappa T_{\mathrm{ref}}} .
$$

The first term in the curly brackets is the classic parabolic departure from a constant temperature, symmetrically centered about the middle of the channel. Obviously the viscosities incorporated in the coupling number $N$ and in the length scale parameter $L$ have an effect on the temperature variation. The expressions in the $a$-term are related to pure translational velocity control of the top plate, the $c$-term includes the influence of pure angular velocity control, and the $b$-term represents the interaction between both.

With the relations shown in (23) it is straightforward to rewrite the temperature in terms of force boundary conditions:

$$
\begin{aligned}
\frac{T(\bar{y})-T_{\text {res }}}{T_{\text {ref }} a / 2}=\bar{y}(1- & \bar{y})-\frac{1}{L^{2}}\left(1-\frac{\cosh (N L[2 \bar{y}-1])}{\cosh (N L)}\right) \\
& -\frac{L^{2} P^{2}}{8 N^{2}}\left(P^{2}-2 P \xi+\xi^{2}\right) \frac{\cosh (2 N L[2 \bar{y}-1])-\cosh (2 N L)}{\sinh ^{2}(N L)} \\
& -\frac{P}{4 N^{2}}(P-\xi)\left(2 \bar{y}-1-\frac{\sinh (N L[2 \bar{y}-1])}{\sinh (N L)}\right) \\
& -\frac{1}{8 L^{2}} \frac{\cosh (2 N L[2 \bar{y}-1])-\cosh (2 N L)}{\cosh ^{2}(N L)}
\end{aligned}
$$




\section{Exploitation of the analytical results}

Consider first the velocity-controlled profiles described by (15). Let us initially assume that $\omega_{0}=0$ and that $N^{2}=0.9$, i.e., strong coupling. Then an S-shaped form of the velocity profile becomes apparent: Figure 2, left. Note that for small values of $L$ as well as for large values the velocity profiles almost coincide with the classical Navier-Stokes while for intermediate values of $L$ the $S$-shape is more pronounced.

In [Ivanova and Vilchevskaya 2016] the particle aspect on a mesoscopic scale was connected to the continuum point of view of a micropolar medium. If we follow the authors' line of reasoning we may argue intuitively and say that for the case when $L$ is very small, i.e., the length of the particles on the mesoscale becomes comparable or greater than the (macroscopic) plate distance, there is no "space for rotation", and the angular velocity vanishes (blue line). From (17) it is clear that the angular velocity can only arise from applied angular velocities at the boundary and is not influenced by the linear velocity boundary conditions in the limit case $L \rightarrow 0$. Furthermore note that in this case the linear velocity is given by the classical Navier-Stokes solution if no-spin boundary conditions are employed; see (17) and Figure 2, left. After normalization with the plate velocity the effect of intermediate length scales on the angular velocity, Figure 2, right, becomes visible: with increasing $L=h / l$, the length of the particles on the mesoscale $l$ compared to the plate distance $h$ allows for more rotation and thus greater magnitudes of the angular velocity occur until the size effect disappears for very large $L$. Observe that for $L=2$ the velocity gradient in the middle of the channel is greater than for an ordinary Navier-Stokes fluid. Furthermore, note that in this case the characteristic
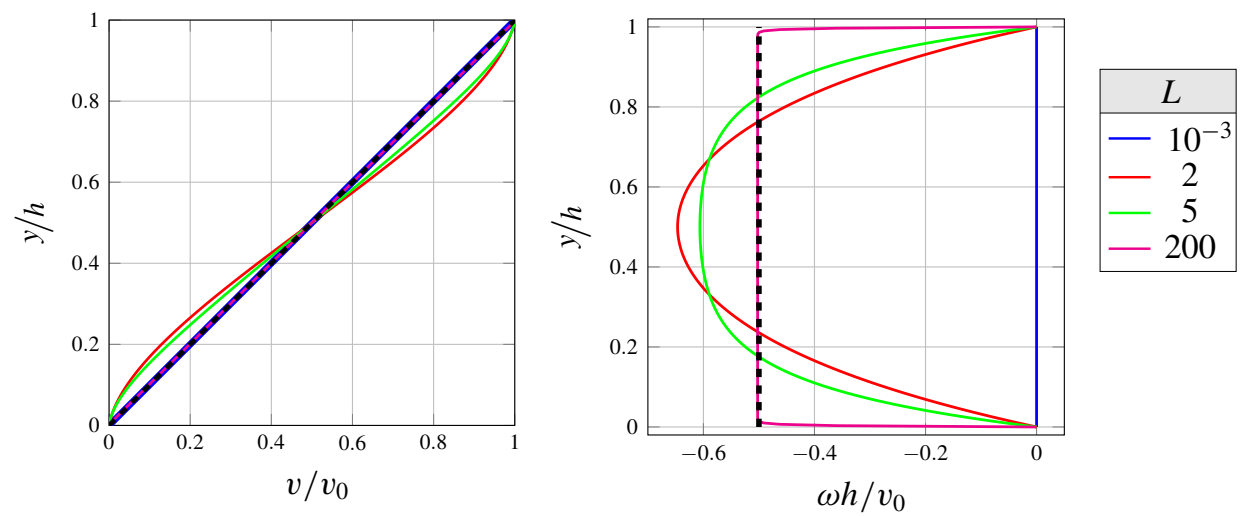

Figure 2. Linear and angular velocity profiles for Couette flow according to the Eringen theory for the velocity-controlled case with no-spin boundary conditions, $\omega_{0}=0$. 

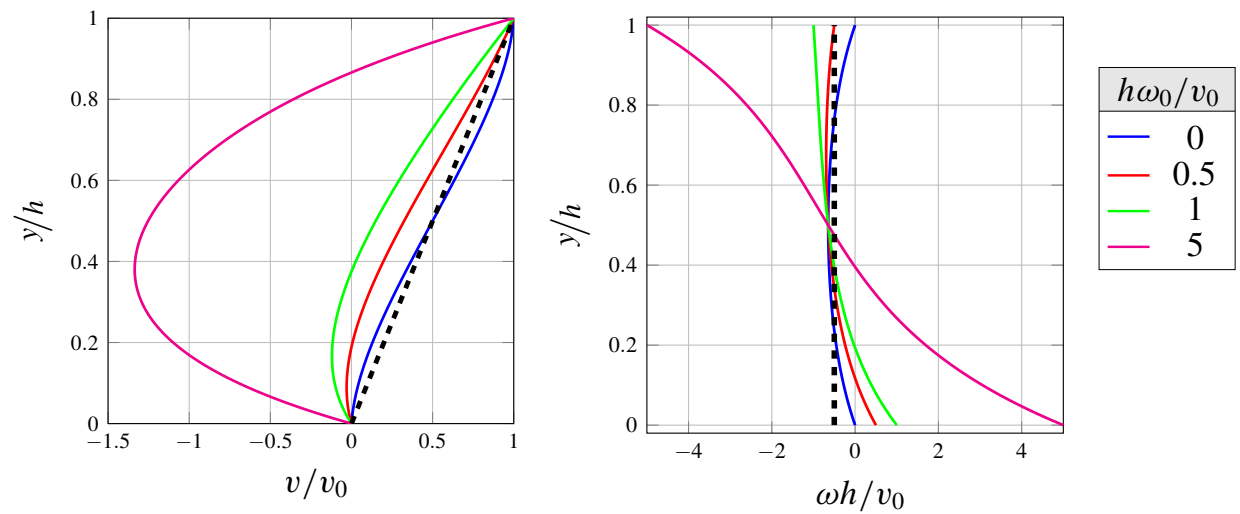

Figure 3. Linear and angular velocity profiles for Couette flow according to the Eringen theory for the velocity-controlled case.

length scale is half of the plate distance. Due to the higher shear rate and the length scale that fits twice into the channel, the fluid particles can rotate more easily and subsequently a greater magnitude of the angular velocity occurs, as can be seen in Figure 2, right. The dashed lines corresponds to a Navier-Stokes fluid, where in Figure 2, right, the vorticity was used because no angular velocity is available for ordinary fluids. ${ }^{3}$

In summary we may say that the plots for the linear velocity have been shown before in the literature [Cowin 1974, p. 323]. However, to the best knowledge of the authors this is surprisingly not so for the angular velocity plot.

In the previous figure we presented solutions for the no-spin boundary condition, i.e., $\omega_{0}=0$. From this case the solutions for $L=2$ are of particular interest and we shall now proceed to employ spin boundary conditions different from zero and keep $L=2$ fixed. In Figure 3 the linear and angular velocity profiles are depicted for different values of the factor $h \omega_{0} / v_{0}$. For sufficiently large values of this factor a significant backflow behavior is observable and the S-shaped velocity profiles turn into more and more wavy curves (Figure 3, left). The angular velocity is no longer an even but an odd function since the boundary conditions at the upper and lower plate only coincide in magnitude but have different signs. As we shall see immediately this behavior changes when we switch to the force-controlled case.

For the case of force control the velocity, normalized by $h t_{x} / \mu$, is illustrated in Figure 4, left. A purely force-controlled case was assumed, i.e., there is no moment per unit area acting on the upper plate, $\mu_{z, \mathrm{t}}=0$, and $N^{2}=0.9$, as before. As to be expected we obtain the bisectrix of ordinary Navier-Stokes flow if the internal length scale is negligible (large values of $L$ ). Intermediate length scales lead to

\footnotetext{
${ }^{3}$ The same mode of presentation will be used in the following plots.
} 

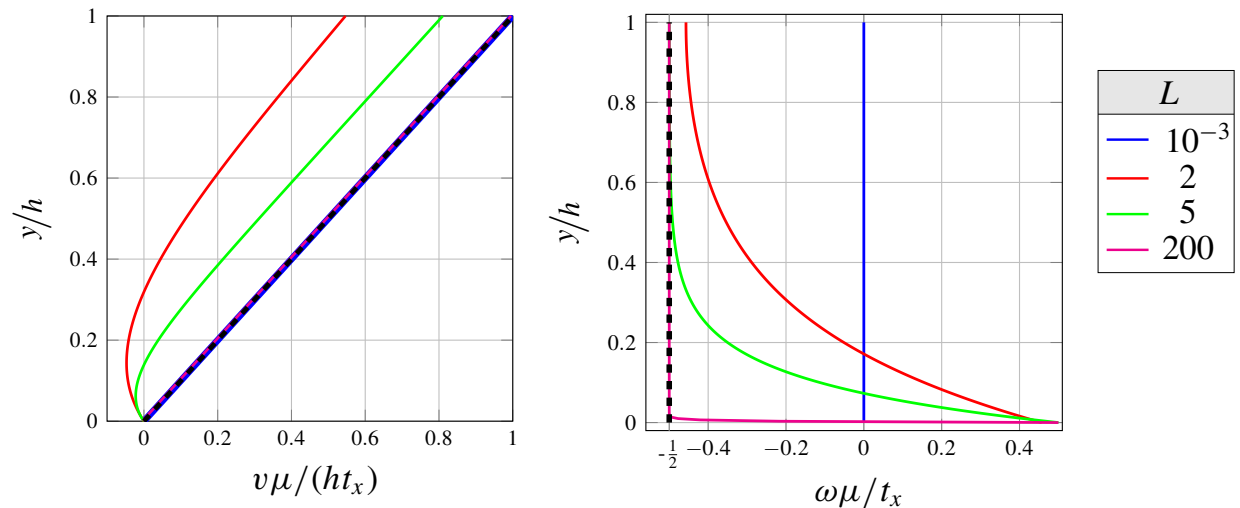

Figure 4. Linear and angular velocity profiles for Couette flow according to the Eringen theory for the force-controlled case with $\mu_{z, \mathrm{t}}=0$.

S-shaped curves showing, most interestingly, backflow behavior. This has been reported recently for nonstationary Couette flow [Magyari et al. 2010], as well as in molecular dynamics simulations of micropolar fluid flow [Haghighi and Asl 2015]. Very small values of $L$ lead again to a straight line but with a much smaller velocity at the top plate which, for equal $t_{x}$, results because of the effectively higher shear viscosity. Also note that for a prescribed force the velocity of the upper plate goes down if the effective viscosity increases, i.e., if we depart from the case of classical Navier-Stokes. This is also intuitively plausible.

It should be mentioned that a similar case of normalization of the velocity is also reported in [Cowin 1974, p. 326], where the case of vanishing angular velocities at the plate boundaries (no slip of the $\omega$-field) was exclusively considered. However, there is a subtle difference from our case if we specialize to $\omega_{0}=0$. According to $(23)_{2}$ a vanishing angular velocity on the top plate corresponds to a nonvanishing moment per unit area, $\mu_{z, \mathrm{t}}=h t_{x} P$. Moment and force are not independent in this case, and one ends up with the relations

$$
\begin{aligned}
\frac{v(\bar{y})}{h t_{x} / \mu} & =\bar{y}-\frac{P}{2}\left(1+\frac{\sinh (N L[2 \bar{y}-1])}{\sinh (N L)}\right), \\
\frac{\omega(\bar{y})}{t_{x} / \mu} & =-\frac{1}{2}+\frac{\cosh (N L[2 \bar{y}-1])}{2 \cosh (N L)} .
\end{aligned}
$$

Thus, we cannot say that Cowin [1974] considers a proper force-controlled case, and in fact he never says he does. Be this as it may, (30) does not show any backflow behavior.

The angular velocity also depends considerably on height, at least for values of $L$ in between the upper and lower bounds: Figure 4, right. If the length scale of 

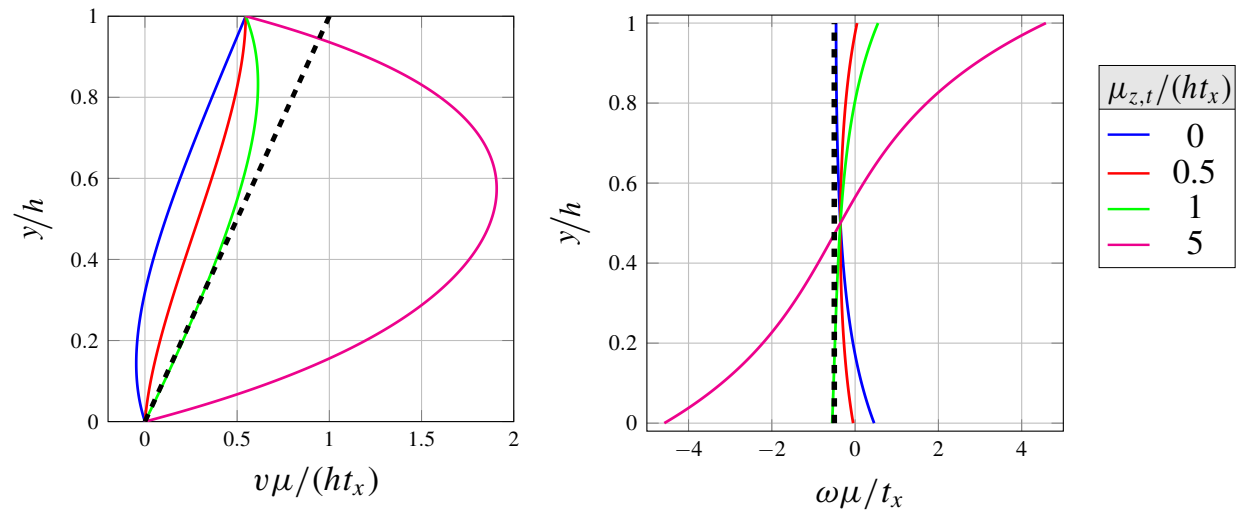

Figure 5. Linear and angular velocity profiles for Couette flow according to the Eringen theory for the force-controlled case.

the micropolar fluid is small compared to the distance of the plates (large values of $L$ ), the angular velocity is completely represented by the vorticity, which is constant: $\omega /\left(t_{x} / h\right)=-\frac{1}{2}$ (magenta curve), as in the case of Navier-Stokes flow (black dashed curve).

The analogue to Figure 3 is shown in Figure 5 (for $L=2$ ). In Figure 5, left, the velocity profiles normalized by the horizontal traction at the upper plate show backflow behavior as well but tend to be more pronounced in the positive $\boldsymbol{e}_{x}$-direction for higher values of the ratio of applied tractions $\mu_{z, \mathrm{t}} /\left(h t_{x}\right)$. In addition, the angular velocity profiles are essentially the same as in the velocity-controlled case but with a different sign so that the angular velocity at the upper plate is now positive.

As far as the temperature profiles of (27) are concerned we first focus on the case where $T_{\text {ref }}=\mu v_{0}^{2} / \kappa$ (in accordance with the normalization used in the classical Navier-Stokes case; see, e.g., [Müller 2014, p. 327]) and $a=\left(\mu_{\mathrm{eff}} / \mu\right)^{2}=$ $(1-P)^{-2}, b=c=0$, i.e., the case of pure translational velocity control. This is shown in Figure 6, left, by using the same parameters as for the plots of Figure 4. Obviously the temperature change in micropolar fluid flow is more pronounced than for a classical Navier-Stokes fluid (dashed line). This is to be expected because of the additional viscosity terms, in particular because of the factor $(1-P)^{-2}$.

Figure 6 , right, concentrates on the case $a=b=0$, i.e., the case of pure angular velocity control. It is now tempting to analyze the situation for $c=1$. However, note that in the limit case $L \rightarrow 0$ (while keeping $\beta+\gamma=$ const.) one has to take into account that the parameter $c$ will not remain constant because

$$
c=\frac{\mu\left(h \omega_{0}\right)^{2}}{\kappa T_{\mathrm{ref}}}=\frac{L^{2}(\beta+\gamma) \omega_{0}^{2}}{\kappa T_{\mathrm{ref}}}
$$



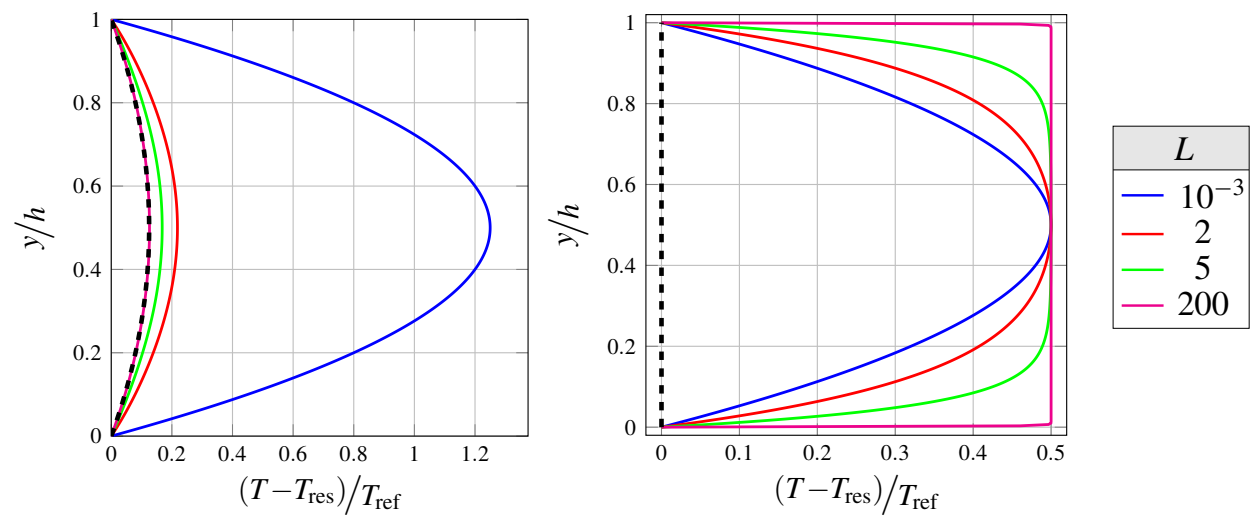

Figure 6. Temperature profiles for Couette flow according to Eringen's micropolar theory.

strongly depends on $L$. Choosing the reference temperature conveniently as $T_{\text {ref }}=$ $(\beta+\gamma) \omega_{0}^{2} / \kappa$ we must put $c=L^{2}$, which balances the otherwise singular term in (27) for very small values of $L$, leading to parabolic profiles. On the other hand, very large values of $L$ imply that the internal length scale is negligible when compared to the plate distance so that the temperature becomes independent from the position between the plates. This leads to a homogeneous temperature profile. Note that this situation does not correspond to an ordinary fluid because there is no angular velocity available in the classical Navier-Stokes case, $\omega \equiv 0$; see Figure 6, right.

\section{Literature review and discussion}

Since the advent of Eringen's theory of micropolar fluids simple flow problems allowing for analytical solutions have continuously been in the focus of interest. Poiseuille flow is most predominantly discussed. In fact, Eringen [1966] gives corresponding solutions for translational and angular velocity in circular pipes, which are later repeated in his textbooks, e.g., [Eringen 2001, §9.7]. The temperature rise due to viscous friction for Poiseuille flow between plates is discussed in [Kazakia and Ariman 1971; Řiha 1975]. A first discussion of the velocity profiles of plane-parallel micropolar Couette flow can be found in [Ariman and Cakmak 1968; Pennington and Cowin 2000; Cowin 1974]. However, these authors constrain themselves to the case of vanishing angular velocity at the plates, $\omega_{0}=0$. Couette flow between rotating cylinders was studied in [Verma and Sehgal 1968], where it was also assumed that the rotational velocities vanish at the cylinder walls.

A discussion of the temperature rise in plane-parallel Couette flow is presented in [Singh 1982]. Although Singh applies his solution to the concrete example of blood flow the general presentation is essentially a mathematical exercise allowing for 
the greatest freedom in the choice of boundary conditions. For example, the effect of microtemperatures is included just like different choices of the top and bottom reservoir temperatures. In a nutshell the final result contains so many abbreviations that it becomes impossible to discern the impact of the various physical parameters. It is for this reason that we have constrained ourselves in this presentation to the most essential ones.

Due to the advent of micro- and nanotechnology the solution to elementary flow problems based on micropolar theory complemented by molecular dynamics simulations is more recently in the focus of research again. Examples are given by [Kucaba-Pietal 2004; Kucaba-Pietal et al. 2009].

Chen et al. [2012] and Ashmawy [2012] present an analysis of transient Couette flow, based on a numerical and a Laplace transform solution, respectively. The angular velocity at the boundaries was once more assumed to be zero.

Hence, it is now high time to talk about how boundary conditions for micropolar media are usually introduced in the literature and, what is even more important, if and how they can be realized experimentally.

It is obvious from the citations we presented so far that it is very popular to postulate so-called "no-slip" conditions or, in other words, that the tangential (translational) velocity of the micropolar fluid is equal to that of the wall and its angular velocity vanishes. However, recall that even in the simpler case of a Navier-Stokes fluid a no-slip condition is by no means as straightforward to realize as we may want to believe at first glance. We simply got so used to it that we rarely question it. However, the scientists who initiated this notion thought differently. From the citations in the nicely written overview on the history of the no-slip condition [Day 1990], we learn that Stokes based his conviction of the applicability of the noslip conditions on certain experiments, in particular one performed by Coulomb, according to which the resistance of water flow against a wall is independent of the surface characteristics (also see [Müller 2007, p. 241]). Hence, it is due to internal friction of the fluid alone, which is consistent with the assumption that the water simply sticks to the wall. This statement was questioned by Maxwell [Brenner 2011] and replaced by the possibility of slip in the case of dilute gases, a phenomenon which becomes ever more important to be taken into account in today's microfluidic systems [Lauga et al. 2007]. Practically speaking, the amount of slip encountered in a particular experimental setup may be controlled (to a certain degree) by polymer or surfactant absorption or other chemical modification of the walls.

Moving now closer to the boundary conditions relevant for micropolar fluids, it should be mentioned that slip of the tangential velocity at a wall was recently considered in order to study the Couette flow of couple stress fluids in [Silber et al. 2007; Alizadeh et al. 2011; Devakar et al. 2014]. The model of a couple stress 
fluid becomes relevant if surface effects occur [Mindlin 1965]. For example, when we add additives to a fluid, the forces present in the fluid oppose the forces of the additives. This opposition leads to a couple force, and hence, a couple stress is induced in the fluid. We may say that this is also an example of materials that require a generalized continuum theory beyond the ordinary Boltzmann-Cauchy approach.

General properties of a couple stress theory for solids were analyzed in [Hadjesfandiari and Dargush 2011]. On the basis of the principle of virtual work and without the use of special constitutive relations the authors showed, e.g., that the moment traction vector is tangent to the surface. However, they restrict their presentation to the equilibrium case, i.e., their results lack generality. Although a couple stress theory for solids has little to do with the theory presented in this work, note that in the case of Couette flow the moment tractions are indeed tangential to the surfaces as can be seen in Table 1 .

Another generalized continuum which, in contrast to couple stress fluids, necessitates us to take the aspect of internal rotational degrees of freedom into account, are nematic liquid crystals. In order to describe their flow behavior the theory proposed by Ericksen and Leslie is frequently applied [Steward 2004]. There are certain differences when compared to the Eringen approach, which is favored in this paper. The microinertia tensor is replaced by the so-called director, often represented by an inextensible one-dimensional digit. It is the continuum representation of the macromolecules liquid crystals are made of. Note that the macromolecules are usually polarized, which is important for realizing the boundary conditions, as we shall discuss shortly. Other "new" quantities in the Ericksen-Leslie theory are the director stress tensor, which replaces the couple stress tensor, and the director production density, which occurs, most importantly, because the spin balance is replaced by what is known as the balance of director force.

In the Ericksen-Leslie theory boundary conditions for the angle of orientation of the director at a wall are required. Two main types of this so-called "strong anchoring" are distinguished [Steward 2004, p. 47], namely homeotropic and homogeneous alignment, where the digits are oriented perpendicular and parallel to the wall, respectively. Note that such conditions are the equivalent of prescribing a vanishing angular velocity at the wall in the case of Eringen's theory of micropolar fluids. But how is this mathematical requirement realized in practice? An answer can be found in [de Gennes and Prost 1995, p. 109]: the surfaces of the walls must be prepared accordingly by "rubbing" thus inducing a certain electric "polarization".

Finally, let us review the situation in the case of micropolar fluids. An excellent summary of the situation, at least from a mathematical point of view, can be found in [Łukaszewicz 1999, p. 30]. First, the author discusses velocity-type boundary 
conditions of the type shown in (11). Specifically he writes

$$
v=v_{\mathrm{b}}, \quad \omega=\omega_{\mathrm{b}},
$$

where it is explicitly mentioned that $\boldsymbol{v}_{\mathrm{b}}$ is the (translational) velocity and $\omega_{\mathrm{b}}$ is the microrotation of points of the solid boundary $b$. The author also refers to $(32)_{1}$ as "physically clear (the viscous fluid sticks to the solid boundary)" and then states that "there is no general agreement as to the type of boundary condition one should set for the field of microrotation" $(32)_{2}$. Indeed, it was mentioned before, and the proper references have been given, that frequently such no-slip boundary conditions are prescribed in micropolar flow problems, i.e., the tangential translational velocity is that of the wall and the angular velocity is either zero or of a fixed value, also characteristic of the wall particles. However, it is usually not discussed how these can be realized physically. In this context recall our remarks and references from above on the no-slip condition for the translational velocity.

However, there are some alternative opinions to the no-slip condition as well. For example, in the early paper by Ahmadi [1976] it is suggested that

$$
\omega=-\frac{1}{2} \frac{\mathrm{d} v}{\mathrm{~d} y} \quad \text { at } y=0, h .
$$

In other words, the angular velocity equals the vorticity, where both are related to fluid and not to wall characteristics. In fact the author says, "In the neighborhood of a rigid boundary the effect of microstructure must be negligible since the suspended particle cannot get closer than their radius to boundary. In the case of blood flow it is observed that the red cells tend not to get very close to the boundary... Therefore, in the neighborhood of the boundary the only rotation is due to fluid shear and therefore, gyration vector must be equal to angular velocity." Clearly, this also means that angular velocity and translational velocity are coupled and no longer independent, which is in disagreement with the beliefs of Eulerian mechanics.

However, this condition was generalized in [Kirwan $1986 ;{ }^{4}$ Kolpashchikov et al. 1983] by modifying it to

$$
\omega_{\mathrm{b}}=\alpha \boldsymbol{w}_{\mathrm{b}},
$$

where through the index " $b$ " reference is made to fluid properties at the boundary. In fact the latter authors say, "The quantity $v$ [i.e., $\omega$ ] in the fluid volume decreases with an increase of the effect of moment stresses between particles upon microrotation, tending to zero, at the absence of external fields. With a decrease of this influence the magnitude $v$ increases and tends to the limit $w=(1 / 2) \operatorname{rot} v$. Thus, a change of $v$ is restricted to limits from 0 to $w$... In the case of complete adhesion,

\footnotetext{
${ }^{4}$ Kirwan addressed the question of boundary conditions even earlier in a less general manner [Kirwan and Newman 1969a; 1969b].
} 
$v_{b}=0$, from where the lower limit of the parameter of boundary conditions $\alpha=0$ follows. With deterioration of the above interaction $v_{b}$ increases up to the value, also determined by moment stresses between the MPF [micropolar fluid] particles. When the moment stresses between the MPF particles and the boundary are sufficiently small, the magnitude $v_{b}$ is determined mainly by the moment stresses between the fluid particles themselves. In the problem under consideration this case is described by the condition"

$$
\left.\frac{\mathrm{d} \omega}{\mathrm{d} y}\right|_{\bar{y}=0 \text { or } 1}=0 .
$$

Continuing, "Equality $\alpha=1$ corresponds to the physical situation, where there are moment stresses and no difference between the angular velocity of particle proper rotation and that of the fluid volume microelement containing it, $w$. Thus, the range of variation of the parameter of boundary conditions is $0 \leq \alpha \leq 1$."

In short, (34) is an attempt to relate angular velocity and the rotation of the translational velocity. We may want to refer to $\alpha=1$ as the fully coupled case and vice versa. In a certain way this equation circumvents the assumption of the principle of independence of both types of velocities and, indeed, quoting Hogan and Henriksen [1989], "No compelling theoretical argument or experimental evidence suggesting a correct value for $S$ [i.e., $\alpha$ ] has been found in the literature."

Nevertheless, if we make use of this boundary condition for $\omega$ the constants $c_{i}$ in (12) can be determined to read

$$
\begin{aligned}
c_{1} & =-\frac{v_{0} P(1-\alpha)}{2\left[(1-P)-\alpha\left(N^{2}-P\right)\right]}, \quad c_{2}=\frac{\left(v_{0} / h\right)\left(1-\alpha N^{2}\right)}{(1-P)-\alpha\left(N^{2}-P\right)}, \\
c_{3 / 4} & =\frac{\left(v_{0} / h\right)(1-\alpha)[1 \mp \tanh (N L)]}{4\left[(1-P)-\alpha\left(N^{2}-P\right)\right]},
\end{aligned}
$$

and the following solutions are obtained:

$$
\begin{aligned}
\frac{v}{v_{0}} & =\frac{\bar{y}\left(1-\alpha N^{2}\right)-\frac{1}{2} P(1-\alpha)(1+\sinh (N L[2 \bar{y}-1]) / \sinh (N L))}{(1-P)-\alpha\left(N^{2}-P\right)}, \\
\frac{\omega}{v_{0} / h} & =\frac{1}{2} \frac{\left(1-\alpha N^{2}\right)-(1-\alpha) \cosh (N L[2 \bar{y}-1]) / \cosh (N L)}{P(1-\alpha)+\left(\alpha N^{2}-1\right)} .
\end{aligned}
$$

In [Kolpashchikov et al. 1983] the question was asked which value for $\alpha$ leads to the condition (35). The answer depends on the boundary value problem under study. In that reference an answer was given for the case of plane-parallel plate flow due to a pressure gradient. Then $\alpha$ can assume arbitrary values between 0 and 1, depending on the choice of micropolar fluid constants $N$ and $L$. If we wish to obtain (35) for our boundary value problem (36)-(37) we must obviously choose $\alpha=1$, i.e., only the case of total coupling prevails. 
More recently an alternative to (33) has been proposed in [Chakraborty and Chakraborty 2008]:

$$
\omega(\bar{y}=1 \text { or } 0)=\left.\beta \frac{\mathrm{d} \omega}{\mathrm{d} y}\right|_{\bar{y}=1 \text { or } 0} .
$$

The coefficient $\beta$, which has the dimension [m], is interpreted as the microrotational slip length and $\left.\frac{\mathrm{d} \omega}{\mathrm{d} y}\right|_{\bar{y}=1 \text { or } 0}$ as (proportional to) the couple stress $\mu_{y z}$ at the walls. This idea has been quantified by molecular dynamics simulations in that reference and continues to be very popular in the most recent literature. The following quote from [Sheikholeslami et al. 2014], where the parameter $s$ is identical to $-\beta$, provides some physical insight into this ansatz: "The case $s=0$ represents concentrated particle flows in which microelements close to the wall are unable to rotate. Other interesting particular cases that have been considered in the literature include $s=0.5$ which represents weak concentrations and the vanishing of the antisymmetric part of the stress tensor and $s=1$ which represents turbulent flow."

Finally, note that molecular dynamics simulations indicate that choosing the noslip condition $\omega_{0}=0$ or a vanishing gradient of the angular velocity (38) seems to be linked to the surface roughness, or to quote Lopez et al. [2016], "The boundary conditions for microrotations... were chosen to be $\omega=0$ at the wall and inlet, and $\frac{\partial \omega}{\partial \eta}=0$ at the outlet and the far field boundary, where $\eta$ is the normal coordinate. The reason to prescribe $\omega=0$ at the inlet and at the wall is to avoid the effects of the wall roughness or perturbations from the free stream entering the boundary layer."

Indeed, such possibilities were anticipated by Łukaszewicz [1999, p. 31], when he talks about the possibility to prescribe forces and moments, as we did in Table 1 . Referring to [Aero et al. 1965] he proposes in a very general manner that at the wall we have

$$
\boldsymbol{n} \cdot \boldsymbol{\mu}=\boldsymbol{A} \cdot(\boldsymbol{\omega}-\boldsymbol{w})
$$

where " $\boldsymbol{A}$ is a matrix with numeric components". We may wish to refer to it as a coupling matrix instead according to the Eulerian credo that angular and translational velocities are independent.

The limit cases $\boldsymbol{A} \rightarrow \infty$ and $\boldsymbol{A} \rightarrow 0$ are treated by putting $\boldsymbol{\omega}=\boldsymbol{w}$, which expresses "a very strong interaction between the fluid particles and the solid boundary the microrotation reduces to the vorticity of points at the boundary caused by its movement". We decided to depict this situation by the cartoon shown in Figure 7. In particular, if the boundary does not move this condition reduces to $\boldsymbol{\omega}=\mathbf{0}$. Despite the nice cartoon shown in the figure, the question as to how to induce an $\omega \neq 0$ at a wall experimentally is unanswered until today and the same holds for the question of the transmission of a prescribed moment density. 


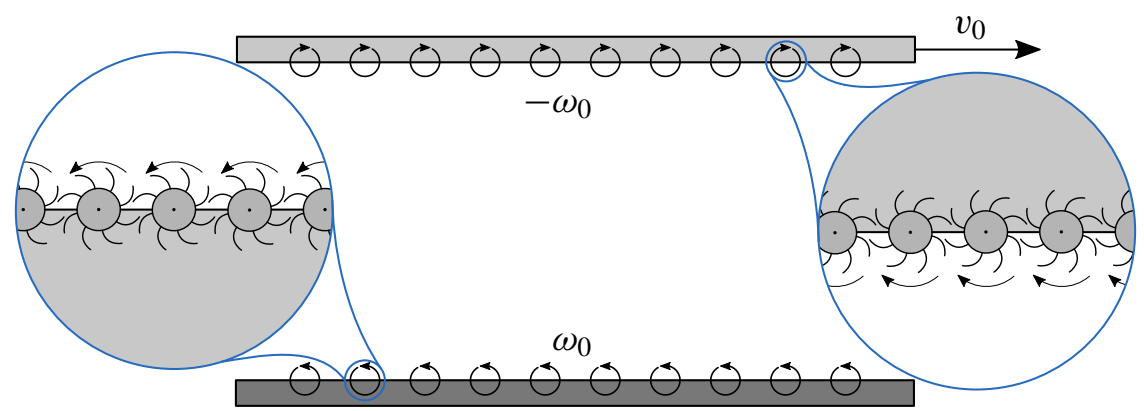

Figure 7. Model concept of imposing the angular velocity field via small paddlewheels.

More generally speaking we may also claim that the question of which boundary conditions to use in the case of theories beyond the Cauchy-Boltzmann continuum is still in need of finding a rational basis for their definition. Eventually the kinetic theory of gases might provide a clue: for the case of ideal gases it was demonstrated in [Barbera et al. 2004] that the missing boundary conditions for the heat flux in an extended thermodynamic theory can be found by minimization of the entropy of the system in question. Clearly, such an approach is much more difficult to realize if fluid matter is concerned.

\section{Conclusions and outlook}

In this paper we provided closed-form solutions of Couette velocity profiles based on Eringen's theory for micropolar fluids. In addition to that the temperature increase due to internal viscous friction was calculated analytically. We analyzed the effects of different boundary conditions and commented on suggestions for these from the literature. Possible corresponding experimental realizations were presented.

\section{Appendix A: Remarks on notation}

For convenience of the reader we present the relevant equations in Cartesian representation. The balances introduced in (1)-(4) read

$$
\begin{aligned}
& 0=\frac{\partial v_{i}}{\partial x_{i}}, \quad \rho_{0} \frac{\mathrm{d} v_{i}}{\mathrm{~d} t}=\frac{\partial \sigma_{j i}}{\partial x_{j}}, \quad \rho_{0} J_{0} \frac{\mathrm{d} \omega_{i}}{\mathrm{~d} t}=\frac{\partial \mu_{j i}}{\partial x_{j}}+\epsilon_{i j k} \sigma_{j k}, \\
& \rho_{0} \frac{\mathrm{d} u}{\mathrm{~d} t}=-\frac{\partial q_{i}}{\partial x_{i}}+\sigma_{i j}\left(\frac{\partial v_{j}}{\partial x_{i}}-\epsilon_{i j k} \omega_{k}\right)+\mu_{i j} \frac{\partial \omega_{j}}{\partial x_{i}},
\end{aligned}
$$


where $\epsilon_{i j k}$ is the Levi-Civita symbol. For the constitutive relations given in (5), (6), and (8) we have

$$
\begin{aligned}
\sigma_{i j} & =-p \delta_{i j}+\mu\left(\frac{\partial v_{i}}{\partial x_{j}}+\frac{\partial v_{j}}{\partial x_{i}}\right)+2 \tau \omega_{k} \epsilon_{k j i}-\tau\left(\frac{\partial v_{i}}{\partial x_{j}}-\frac{\partial v_{j}}{\partial x_{i}}\right), \\
\mu_{i j} & =\alpha \frac{\partial \omega_{k}}{\partial x_{k}} \delta_{i j}+\beta\left(\frac{\partial \omega_{i}}{\partial x_{j}}+\frac{\partial \omega_{j}}{\partial x_{i}}\right)-\gamma\left(\frac{\partial \omega_{i}}{\partial x_{j}}-\frac{\partial \omega_{j}}{\partial x_{i}}\right), \\
q_{i} & =-\kappa \frac{\partial T}{\partial x_{i}}+\lambda \frac{\partial \omega_{k}}{\partial x_{j}} \epsilon_{i j k},
\end{aligned}
$$

where $\delta_{i j}$ is the Kronecker symbol and $h_{i}=\omega_{i}-\frac{1}{2} \epsilon_{i j k} \frac{\partial v_{k}}{\partial x_{j}}$ was used. Finally, with the aid of the relations

$$
\begin{aligned}
& \nabla \cdot(\boldsymbol{h} \times \mathbf{I})=\frac{\partial}{\partial x_{i}} \boldsymbol{e}_{i} \cdot\left(h_{j} \boldsymbol{e}_{j} \times \boldsymbol{e}_{k} \otimes \boldsymbol{e}_{k}\right)=\frac{\partial h_{j}}{\partial x_{i}} \epsilon_{j k i} \boldsymbol{e}_{k}=\nabla \times \boldsymbol{h}, \\
& \boldsymbol{\sigma}_{\times}=2 \tau(\boldsymbol{h} \times \mathbf{I})_{\times}=2 \tau h_{i}\left(\boldsymbol{e}_{i} \times \boldsymbol{e}_{j}\right) \times \boldsymbol{e}_{j}=2 \tau h_{i} \epsilon_{i j k} \epsilon_{k j m} \boldsymbol{e}_{m}=-4 \tau \boldsymbol{h}, \\
& 2 \boldsymbol{w} \times \mathbf{I}=(\nabla \otimes \boldsymbol{v})^{\top}-\nabla \otimes \boldsymbol{v}, \\
& \nabla \times(\nabla \times \boldsymbol{v})=\nabla(\nabla \cdot \boldsymbol{v})-\Delta \boldsymbol{v}, \\
& \boldsymbol{e}_{i} \cdot \boldsymbol{e}_{j}=\delta_{i j}, \quad \boldsymbol{e}_{i} \times \boldsymbol{e}_{j}=\epsilon_{i j k} \boldsymbol{e}_{k}, \quad \epsilon_{i j k} \epsilon_{j k m}=2 \delta_{i m},
\end{aligned}
$$

where $\boldsymbol{e}_{i}$ are the base vectors, the balances of linear momentum and spin in (7) are obtained taking into account the incompressibility condition:

$$
\begin{aligned}
\rho_{0} \frac{\mathrm{d} v_{i}}{\mathrm{~d} t} & =-\frac{\partial p}{\partial x_{i}}+(\mu+\tau) \frac{\partial^{2} v_{i}}{\partial x_{k} \partial x_{k}}+2 \tau \frac{\partial \omega_{k}}{\partial x_{j}} \epsilon_{i j k}, \\
\rho_{0} J_{0} \frac{\mathrm{d} \omega_{i}}{\mathrm{~d} t} & =(\alpha+\beta-\gamma) \frac{\partial^{2} \omega_{j}}{\partial x_{i} \partial x_{j}}+(\beta+\gamma) \frac{\partial^{2} \omega_{i}}{\partial x_{k} \partial x_{k}}-4 \tau \omega_{i}+2 \tau \frac{\partial v_{k}}{\partial x_{j}} \epsilon_{i j k} .
\end{aligned}
$$

\section{Appendix B: Solution of the coupled system of partial differential equations}

In order to solve the boundary value problem shown in (10) the equations are recast into a system of first-order differential equations:

$$
\frac{\mathrm{d} \boldsymbol{u}}{\mathrm{d} y}=\boldsymbol{C} \cdot \boldsymbol{u} \quad \text { with } \quad \boldsymbol{u}=\left[\begin{array}{c}
v \\
v^{\prime} \\
\omega \\
\omega^{\prime}
\end{array}\right] \text { and } \boldsymbol{C}=\left[\begin{array}{cccc}
0 & 1 & 0 & 0 \\
0 & 0 & 0 & -\frac{2 \tau}{\mu+\tau} \\
0 & 0 & 0 & 1 \\
0 & \frac{2 \tau}{\beta+\gamma} & \frac{4 \tau}{\beta+\gamma} & 0
\end{array}\right] .
$$

By employing the standard exponential ansatz for $\boldsymbol{u}$ we obtain an eigenvalue problem

$$
\boldsymbol{u}=\exp (\lambda y) \boldsymbol{r} \quad \Longrightarrow \quad \lambda \boldsymbol{r}=\boldsymbol{C} \cdot \boldsymbol{r}
$$


If $\boldsymbol{C}$ has four linear independent eigenvectors $\boldsymbol{r}_{i}$, then the general solution to the system (44) is given by

$$
\boldsymbol{u}=\sum_{i=1}^{4} c_{i} \boldsymbol{u}_{i}=\sum_{i=1}^{4} c_{i} \exp \left(\lambda_{i} y\right) \boldsymbol{r}_{i},
$$

where $\lambda_{i}$ are the eigenvalues of $\boldsymbol{C}$ and $c_{i}$ are arbitrary constants. However, in our case $\boldsymbol{C}$ does not have four independent eigenvectors:

$$
\begin{aligned}
& \lambda_{1 / 2}=0, \quad \lambda_{3 / 4}= \pm \frac{2 N L}{h}, \boldsymbol{r}_{1}=\left[\begin{array}{l}
1 \\
0 \\
0 \\
0
\end{array}\right], \quad \boldsymbol{r}_{3 / 4}=\left[\begin{array}{c}
\mp N h / L \\
-2 N^{2} \\
1 \\
\pm 2 N L / h
\end{array}\right], \\
& \text { with } \quad N=\sqrt{\frac{\tau}{\mu+\tau}}, \quad L=\frac{h}{l}, \quad \text { and } \quad l=\sqrt{\frac{\beta+\gamma}{\mu} .}
\end{aligned}
$$

The (double) eigenvalue $\lambda_{1 / 2}$ is incomplete which means that only one linear independent eigenvector $\boldsymbol{r}_{1}$ can be found. Hence, an independent solution $\boldsymbol{u}_{2}$ needs to be constructed by setting [Walter 1998, p. 183]

$$
\boldsymbol{u}_{2}=\exp \left(\lambda_{1} y\right)\left(y \boldsymbol{r}_{1}+\boldsymbol{r}_{2}\right), \quad\left(\boldsymbol{C}-\lambda_{1} \mathbf{1}\right) \cdot \boldsymbol{r}_{2}=\boldsymbol{r}_{1} \quad \Longrightarrow \quad \boldsymbol{r}_{2}=\left[\begin{array}{llll}
0 & 1 & -\frac{1}{2} & 0
\end{array}\right]^{\top},
$$

where $\mathbf{1}$ is the unit matrix. Then, the first and third components of the linear combination of $\boldsymbol{u}_{i}$ are the wanted solutions.

\section{References}

[Aero et al. 1965] E. L. Aero, A. N. Bulygin, and E. V. Kuvshinskii, "Asymmetric hydromechanics", J. Appl. Math. Mech. 29:2 (1965), 333-346.

[Ahmadi 1976] G. Ahmadi, "Self-similar solution of imcompressible micropolar boundary layer flow over a semi-infinite plate”, Internat. J. Engrg. Sci. 14:7 (1976), 639-646.

[Alizadeh et al. 2011] M. Alizadeh, G. Silber, and A. Ghasemi Nejad, "A continuum mechanical gradient theory with an application to fully developed turbulent flows", J. Disper. Sci. Technol. 32:2 (2011), 185-192.

[Ariman and Cakmak 1968] T. Ariman and A. S. Cakmak, "Some basic viscous flows in micropolar fluids", Rheol. Acta 7:3 (1968), 236-242.

[Ashmawy 2012] E. A. Ashmawy, "Unsteady Couette flow of a micropolar fluid with slip", Meccanica 47:1 (2012), 85-94.

[Barbera et al. 2004] E. Barbera, I. Müller, D. Reitebuch, and N.-R. Zhao, "Determination of boundary conditions in extended thermodynamics via fluctuation theory", Contin. Mech. Thermodyn. 16:5 (2004), 411-425.

[Brenner 2011] H. Brenner, "Beyond the no-slip boundary condition", Phys. Rev. E 84:4 (2011), 046309. 
[Chakraborty and Chakraborty 2008] D. Chakraborty and S. Chakraborty, "Towards a generalization of hydrodynamic boundary conditions for flows of fluids with homogeneous internally rotating structures", Phys. Lett. A 372:33 (2008), 5462-5466.

[Chapman and Cowling 1952] S. Chapman and T. G. Cowling, The mathematical theory of nonuniform gases: an account of the kinetic theory of viscosity, thermal conduction and diffusion in gases, 2nd ed., Cambridge University, 1952.

[Chen et al. 2012] J. Chen, C. Liang, and J. D. Lee, "Numerical simulation for unsteady compressible micropolar fluid flow”, Comput. Fluids 66 (2012), 1-9.

[Condiff and Dahler 1964] D. W. Condiff and J. S. Dahler, "Fluid mechanical aspects of antisymmetric stress", Phys. Fluids 7 (1964), 842-854.

[Cowin 1974] S. C. Cowin, "The theory of polar fluids", Adv. Appl. Mech. 14 (1974), 279-347.

[Day 1990] M. A. Day, "The no-slip condition of fluid dynamics”, Erkenntnis 33:3 (1990), 285-296.

[Devakar et al. 2014] M. Devakar, D. Sreenivasu, and B. Shankar, "Analytical solutions of couple stress fluid flows with slip boundary conditions”, Alexandria Engrg. J. 53:3 (2014), 723-730.

[Eringen 1966] A. C. Eringen, "Theory of micropolar fluids”, J. Math. Mech. 16 (1966), 1-18.

[Eringen 1999] A. C. Eringen, Microcontinuum field theories, I: Foundations and solids, Springer, 1999.

[Eringen 2001] A. C. Eringen, Microcontinuum field theories, II: Fluent media, Springer, 2001.

[Gauthier and Jahsman 1975] R. D. Gauthier and W. E. Jahsman, "A quest for micropolar elastic constants", J. Appl. Mech. 42:2 (1975), 369-374.

[de Gennes and Prost 1995] P. G. de Gennes and J. Prost, The physics of liquid crystals, 2nd ed., Oxford University, 1995.

[Hadjesfandiari and Dargush 2011] A. R. Hadjesfandiari and G. F. Dargush, "Couple stress theory for solids", Int. J. Solids Struct. 48:18 (2011), 2496-2510.

[Haghighi and Asl 2015] A. R. Haghighi and M. S. Asl, "Mathematical modeling of micropolar fluid flow through an overlapping arterial stenosis", Int. J. Biomath. 8:4 (2015), 1550056.

[Hogan and Henriksen 1989] H. A. Hogan and M. Henriksen, "An evaluation of a micropolar model for blood flow through an idealized stenosis", J. Biomech. 22:3 (1989), 211-218.

[Ivanova and Vilchevskaya 2016] E. A. Ivanova and E. N. Vilchevskaya, "Micropolar continuum in spatial description”, Contin. Mech. Thermodyn. 28:6 (2016), 1759-1780.

[Kazakia and Ariman 1971] Y. Kazakia and T. Ariman, "Heat-conducting micropolar fluids", Rheol. Acta 10:3 (1971), 319-325.

[Kirwan 1986] A. D. Kirwan, Jr., "Boundary conditions for micropolar fluids", Int. J. Engrg. Sci. 24:7 (1986), 1237-1242.

[Kirwan and Newman 1969a] A. D. Kirwan, Jr. and N. Newman, "Plane flow of a fluid containing rigid structures", Int. J. Engrg. Sci. 7:8 (1969), 883-893.

[Kirwan and Newman 1969b] A. D. Kirwan, Jr. and N. Newman, "Simple flow of a fluid containing deformable structures", Int. J. Engrg. Sci. 7:10 (1969), 1067-1078.

[Kolpashchikov et al. 1983] V. L. Kolpashchikov, N. P. Migun, and P. P. Prokhorenko, "Experimental determination of material micropolar fluid constants", Int. J. Engrg. Sci. 21:4 (1983), 405-411.

[Kucaba-Pietal 2004] A. Kucaba-Pietal, "Microchannels flow modelling with the micropolar fluid theory", B. Pol. Acad. Sci. Tech. 52:3 (2004), 209-214.

[Kucaba-Pietal et al. 2009] A. Kucaba-Pietal, Z. Walenta, and Z. Peradzyński, "Molecular dynamics computer simulation of water flows in nanochannels", B. Pol. Acad. Sci. Tech. 57:1 (2009), 55-61. 
[Lauga et al. 2007] E. Lauga, M. Brenner, and H. Stone, "Microfluidics: the no-slip boundary condition", Chapter 19, pp. 1219-1240 in Springer handbook of experimental fluid mechanics, edited by C. Tropea et al., Springer, 2007.

[Lopez et al. 2016] M. Lopez, J. Chen, and V. A. Palochko, "A multiscale study of the boundary layer development for microfluidic system", Mol. Simulat. 42:16 (2016), 1370-1378.

[Łukaszewicz 1999] G. Łukaszewicz, Micropolar fluids: theory and applications, Birkhäuser, 1999. [Magyari et al. 2010] E. Magyari, I. Pop, and P. P. Valkó, "Stokes' first problem for micropolar fluids”, Fluid Dyn. Res. 42:2 (2010), 025503.

[Mindlin 1965] R. D. Mindlin, "Second gradient of strain and surface-tension in linear elasticity", Int. J. Solids Struct. 1:4 (1965), 417-438.

[Müller 2007] I. Müller, A history of thermodynamics: the doctrine of energy and entropy, Springer, 2007.

[Müller 2014] W. H. Müller, An expedition to continuum theory, Solid Mechanics and its Applications 210, Springer, 2014.

[Pennington and Cowin 2000] C. J. Pennington and S. C. Cowin, "Couette flow of a polar fluid", $T$. Soc. Rheol. 13:3 (2000), 387-403.

[Sheikholeslami et al. 2014] M. Sheikholeslami, M. Hatami, and D. D. Ganji, "Micropolar fluid flow and heat transfer in a permeable channel using analytical method", J. Mol. Liq. 194 (2014), 30-36.

[Silber et al. 2007] G. Silber, U. Janoske, M. Alizadeh, and G. Benderoth, "An application of a gradient theory with dissipative boundary conditions to fully developed turbulent flow", J. Fluids Engrg. 129:5 (2007), 643-651.

[Singh 1982] K. Singh, "Couette flow of microthermopolar fluids between two parallel plates", Acta Mech. 43:1-2 (1982), 1-13.

[Steward 2004] I. W. Steward, The static and dynamic continuum theory of liquid crystals: a mathematical introduction, Taylor \& Francis, 2004.

[Verma and Sehgal 1968] P. D. S. Verma and M. M. Sehgal, "Couette flow of micropolar fluids", Int. J. Engrg. Sci. 6:4 (1968), 233-238.

[Řiha 1975] P. Řiha, "On the theory of heat-conducting micropolar fluids with microtemperatures", Acta Mech. 23:1-2 (1975), 1-8.

[Walter 1998] W. Walter, Ordinary differential equations, Graduate Texts in Math. 182, Springer, 1998.

[Zhilin 2012] P. A. Zhilin, Rational continuum mechanics, Saint Petersburg Polytechnic University, 2012. In Russian.

[Zueger and Lakes 2016] Z. Zueger and R. S. Lakes, "Experimental Cosserat elasticity in open-cell polymer foam”, Philos. Mag. 96:2 (2016), 93-111.

Received 8 Jun 2018. Revised 16 Aug 2018. Accepted 20 Sep 2018.

WILHELM RICKERT: rickert@tu-berlin.de

Institut für Mechanik, Fachgebiet Kontinuumsmechanik und Materialtheorie,

Technische Universität Berlin, Berlin, Germany

ELENA N. VILCHEVSKAYA: vilchevska@gmail.com

Applied Research Laboratory, Institute for Problems in Mechanical Engineering,

Russian Academy of Sciences, Saint Petersburg, Russia

WOLFGANG H. MÜLLER: wolfgang.h.mueller@tu-berlin.de

Institut für Mechanik, Fachgebiet Kontinuumsmechanik und Materialtheorie,

Technische Universität Berlin, Berlin, Germany 
EDITORIAL BOARD

ANTONIO CARCATERRA

ERIC A. CARLEN

FRANCESCO DELL'ISOLA

RAFFAELE ESPOSITO

ALBERT FANNJIANG

Gilles A. FrancFort

Pierangelo Marcati

JEAN-JACQUES MARIGO

PETER A. MARKOWICH

MARTIN OSTOJA-STARZEWSKI

PIERRE SEPPECHER

DAVID J. STEIGMANN

PAUL STEINMANN

PierRe M. SuQueT

MANAGING EDITORS

MICOL AMAR

ANGELA MADEO

MARTIN OSTOJA-STARZEWSKI

\section{ADVISORY BOARD}

ADNAN AKAY

Holm AltenbaCH

MICOL AMAR

HARM ASKES

TEODOR ATANACKOVIĆ

VICTOR BERDICHEVSKY

GuY BouchitTÉ

ANDREA BRAIDES

ROBERTO CAMASSA

MAURO CARFORE

ERIC DARVE

FELIX DARVE

ANNA DE MASI

Gianpietro Del Piero

Emmanuele Di Benedetto

VICTOR A. EREMEYEV

BERNOLD FIEDLER

IRENE M. GAMBA

DAVID Y. GAO

SERGEy GaVRILYUK

Timothy J. HeAley

DOMINIQUE JEULIN

ROgER E. KHAYAT

CORRADO LATTANZIO

ROBERT P. LIPTON

ANGELO LUONGO

ANGEla MADEO

JUAN J. MANFREDI

CARlo MARCHIORO

ANIL MISRA

ROBERTO NATALINI

PATRIZIO NEFF

Thomas J. Pence

ANDREY PIATNITSKI

ERRICO PRESUTtI

MARIO PUlVIRENTI

Lucio Russo

Miguel A. F. SANJUAN

PATRICK SElvaduraI

MIROSLAV ŠILHAVÝ

GUIDO SWEERS

ANTOINETTE TORDESILLAS

LEV TRUSKINOVSKY

JUAN J. L. VELÁZQUEZ

VINCENZO VESPRI

ANGELO VULPIANI msp.org/memocs

Università di Roma "La Sapienza", Italia

Rutgers University, USA

(CO-CHAIR) Università di Roma "La Sapienza", Italia

(TREASURER) Università dell'Aquila, Italia

University of California at Davis, USA

(CO-CHAIR) Université Paris-Nord, France

Università dell' Aquila, Italy

École Polytechnique, France

DAMTP Cambridge, UK, and University of Vienna, Austria

(CHAIR MANAGING EDITOR) Univ. of Illinois at Urbana-Champaign, USA

Université du Sud Toulon-Var, France

University of California at Berkeley, USA

Universität Erlangen-Nürnberg, Germany

LMA CNRS Marseille, France

Università di Roma "La Sapienza", Italia

Université de Lyon-INSA (Institut National des Sciences Appliquées), France (CHAIR MANAGING EDITOR) Univ. of Illinois at Urbana-Champaign, USA

Carnegie Mellon University, USA, and Bilkent University, Turkey

Otto-von-Guericke-Universität Magdeburg, Germany

Università di Roma "La Sapienza”, Italia

University of Sheffield, UK

University of Novi Sad, Serbia

Wayne State University, USA

Université du Sud Toulon-Var, France

Università di Roma Tor Vergata, Italia

University of North Carolina at Chapel Hill, USA

Università di Pavia, Italia

Stanford University, USA

Institut Polytechnique de Grenoble, France

Università dell' Aquila, Italia

Università di Ferrara and International Research Center MEMOCS, Italia

Vanderbilt University, USA

Gdansk University of Technology, Poland

Freie Universität Berlin, Germany

University of Texas at Austin, USA

Federation University and Australian National University, Australia

Université Aix-Marseille, France

Cornell University, USA

École des Mines, France

University of Western Ontario, Canada

Università dell' Aquila, Italy

Louisiana State University, USA

Università dell'Aquila, Italia

Université de Lyon-INSA (Institut National des Sciences Appliquées), France University of Pittsburgh, USA

Università di Roma "La Sapienza", Italia

University of Kansas, USA

Istituto per le Applicazioni del Calcolo "M. Picone", Italy

Universität Duisburg-Essen, Germany

Michigan State University, USA

Narvik University College, Norway, Russia

Università di Roma Tor Vergata, Italy

Università di Roma “La Sapienza”, Italia

Università di Roma “Tor Vergata", Italia

Universidad Rey Juan Carlos, Madrid, Spain

McGill University, Canada

Academy of Sciences of the Czech Republic

Universität zu Köln, Germany

University of Melbourne, Australia

École Polytechnique, France

Bonn University, Germany

Università di Firenze, Italia

Università di Roma La Sapienza, Italia

MEMOCS (ISSN 2325-3444 electronic, 2326-7186 printed) is a journal of the International Research Center for the Mathematics and Mechanics of Complex Systems at the Università dell'Aquila, Italy.

Cover image: "Tangle” by $\odot$ John Horigan; produced using the Context Free program (contextfreeart.org).

PUBLISHED BY

7 mathematical sciences publishers

nonprofit scientific publishing

http://msp.org/

(C) 2019 Mathematical Sciences Publishers 
Mathematics and Mechanics of Complex Systems vol. 7 no. 1

Homogenization of nonlinear inextensible pantographic structures by $\Gamma$-convergence

Jean-Jacques Alibert and Alessandro Della Corte A note on Couette flow of micropolar fluids according to Eringen's theory

Wilhelm Rickert, Elena N. Vilchevskaya and Wolfgang H.

Müller

Analytical solutions for the natural frequencies of rectangular symmetric angle-ply laminated plates

Florence Browning and Harm Askes

On the blocking limit of steady-state flow of Herschel-Bulkley fluid

Farid Messelmi

Continuum theory for mechanical metamaterials with a cubic lattice substructure

Simon R. Eugster, Francesco dell' Isola and David J.

Steigmann

MEMOCS is a journal of the International Research Center for the Mathematics and Mechanics of Complex Systems at the Università dell' Aquila, Italy.

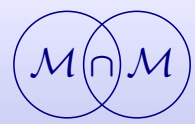

